\title{
Optimization of Assignment of Rapid Train Stops: Example of the JR Nambu Line*
}

\author{
Keiya OKADA** and Ryuhei MIYASHIRO ${ }^{* * *}$ \\ ** Graduate School of Engineering, Tokyo University of Agriculture and Technology, \\ Naka-cho, Koganei, Tokyo 184-8588, Japan \\ E-mail: $50011646109 @$ st.tuat.ac.jp \\ *** Institute of Engineering, Tokyo University of Agriculture and Technology, \\ Naka-cho, Koganei, Tokyo 184-8588, Japan \\ E-mail: r-miya@cc.tuat.ac.jp
}

\begin{abstract}
On many railway lines, not only local trains but also rapid trains are operated. Operating rapid trains can improve the convenience of the passengers, but that outcome strongly depends on the assignment of rapid train stops, the associated timetable, and the number of incoming and outgoing passengers at stops on the way. In particular, to fix assignment of rapid train stops is of great importance. Some previous studies have been conducted to determine optimal assignment of rapid train stops; however, they have relied on approximation methods such as genetic algorithms and local searches. In this study, an enumeration-based algorithm to find optimal assignment of rapid train stops is proposed. Taking actual origin-destination (OD) data of the JR Nambu line, which is a medium-scaled line with 25 stations in Japan, as an example, the proposed algorithm determines optimal assignment of rapid train stops and the associated timetable.
\end{abstract}

Key words : Railway, Scheduling, Timetable, Simulation, Algorithm, Transportation Engineering, Engineering Optimization

\section{Introduction}

Several railway companies operate rapid trains, which skip some stations, for faster transportation. For good rapid train service, assignment of rapid train stops is a key factor. If the assignment is inappropriate, operating rapid trains would increase total travel time for passengers. To obtain the assignment that minimizes total travel time for passengers, previous researches employing approximation algorithms, such as genetic algorithms or local searches. Contrary to these researches, we propose an enumeration-based algorithm to obtain optimal assignment of rapid train stops. In addition, using actual origin-destination (OD) data of the JR Nambu line, we perform computational experiments to show that the proposed algorithm works well for a medium-scaled railway line.

\section{Problem}

In this paper, a railway line that satisfies the following conditions is considered. In addition, the railway line is assumed to be medium-sized, i.e., a line consisting of less than 30 stations.

Conditions for the railway line

(1) Neither branching nor confluent lines exist in the railway line.

(2) All intervals between stations are double-tracked.

( 3 ) For all trains, the two stations at the both end of the line are the terminal stations. 
Rapid trains provide rapid service by skipping some stations, whereas local trains stop at all stations.

Conditions for rapid trains

( 4 ) In the railway line, only rapid and local trains are operated; no express, limited express, etc. are operated.

( 5 ) All rapid trains have same assignment of rapid train stops.

The duration between the terminal stations by a rapid train is shorter than that by a local train. Accordingly, a rapid train passes a local train during its operation. For passing, the following conditions are assumed.

Conditions for passing

( 6 ) Each rapid train passes a local train exactly once during its operation.

( 7 ) Passing is allowed only at fixed stations.

( 8 ) A rapid train stops at stations where the train does pass a local train.

Although a rapid train without passing any local train is imaginable, it does not make sense in improving transportation service, and hence we do not deal with such rapid trains here. In addition, for a medium-scaled railway line a rapid train passing local trains more than once is impractical. Hence, in this study the number of passing by each rapid train is set to one, as stated in Condition (6).

A station where a rapid train can pass a local train is called a possible passing station. On a double-tracked line, a station normally has two platforms, of which one is for inbound trains, and the other is for outbound trains. However to allow passing, a station must have multiple platforms for one direction. A few stations provide such a facility: a waiting lane. Some of possible passing stations have multiple platforms only for inbound or only for outbound trains. In such stations, passing for a certain direction is possible but passing for the other direction is impossible.

For a given timetable, a station where a rapid train does pass a local train is called a passing station. In some railway lines, rapid trains do not stop a passing station. However, in most researches on this area, rapid trains are assumed to stop at a passing station, for convenience of passengers making transfer there. It should be noted that the passing station for inbound trains can be different from that for outbound ones; rapid trains for each direction stop both passing stations if the stations are different.

Conditions for train operation are set as below.

Conditions for train operation

(9) The number of rapid trains operated per hour is at least one, and is at most that of local trains.

(10) For local trains, excluding the waiting time at passing stations, the duration of travel between a pair of stations is a given constant.

(11) For each pair of stations at which rapid trains stop, the duration between them by any rapid train is $m-k \times 1$ minutes, where $m$ is the duration between them by a local train without being passed, and $k$ is the number of stations being skipped between them.

(12) At each station, the interval between two consecutive trains is at least two minutes.

(13) At the terminal stations, the order of departure of local and rapid trains is equitable.

On real railway lines, local trains sometimes travel between a pair of stations in different durations to adjust the interval between the preceding and subsequent trains. However, in most studies, the duration of travel by local trains between a pair of stations is identical, excluding the time of waiting at passing stations, which is described later. 
About the duration of travel by rapid trains between two stations, Condition (11) is a good approximation for actual railway lines. It has been used in several former studies, and we simply employ it here again.

To prevent train collisions, the time interval between two consecutive trains is at least two minutes. Accordingly, at any station, no train arrives there less than two minutes after the preceding one arrived there, and none departs there less than two minutes after the preceding one left there.

The word "equitable" in Condition (13) should be explained. For example, when two rapid trains and four local ones depart from a terminal station per hour, the order of "rapid, rapid, local, local, local, local" is definitely poor service for passengers. Other sequences such as "local, rapid, local, local, rapid, local" is desirable. Condition (13) implies such restrictions.

Conditions for the timetable and passengers are set as below.

Conditions for the timetable and passengers

(14) Only daytime hours, i.e., 9:00-17:00, which exclude rush hours, are examined.

(15) Each station has a timetable repeating a same pattern in each one-hour time zone, 9:00-10:00, 10:00-11:00, .., and 16:00-17:00.

(16) Passengers arrive at their origin station uniformly during daytime.

(17) Passengers manage themselves to take the earliest train(s) arriving at their destination station.

The proposed timetable will be the same for every 60 minutes, which is easily understandable for passengers and advance the ease of traffic control.

When the number of operated trains is few, some smart passengers would remember the timetable and arrive at their origin station immediately before departure time. However, in this study, passengers are assumed to come to the station uniformly. Consequently, for example, at a station where no rapid train stops and local trains depart at 10-minute intervals, a passenger waits there five minutes on average. In addition, although some passengers hating transfers would take only a local train, passengers in this study are assumed to make the quickest trip. Conditions (16) and (17) are also employed in many studies.

Under these conditions listed above and using OD data of passengers, the total travel time of passengers is defined. A travel time of a passenger is defined as the duration between the time when one arrives at the origin station and that when one arrives at the destination station.

The objective of the problem is to create assignment of rapid train stops and the associated timetable that minimize the total travel time of passengers.

\section{Previous Researches}

Some studies have been conducted to determine optimal assignment of rapid train stops. Suzuki and Ishizuka ${ }^{(1)}$ applied dynamic programming to minimize the train ride duration. To improve this method, Futami, et al. ${ }^{(2)}$ used dynamic programming and genetic algorithms. Although they minimized the train ride duration, they provided no consideration of the waiting time of passengers at origin stations. Matsumura ${ }^{(3)}$ considered the waiting time at the passing station and that at origin stations. Incorporating the time at passing stations, he assigned rapid train stops and determined the services of rapid and local trains using a genetic algorithm. Katori, et al. ${ }^{(4)}$ also applied a genetic algorithm to assign rapid train stops. Hiroto ${ }^{(5)}$ used local searches to assign rapid train stops for five railway lines in Japan.

\section{Algorithm}

As mentioned in the previous section, most studies relied on approximation methods such as genetic algorithms and local searches. In contrast, this study proposes an enumerationbased algorithm to find optimal assignment of rapid train stops by examining every possible 


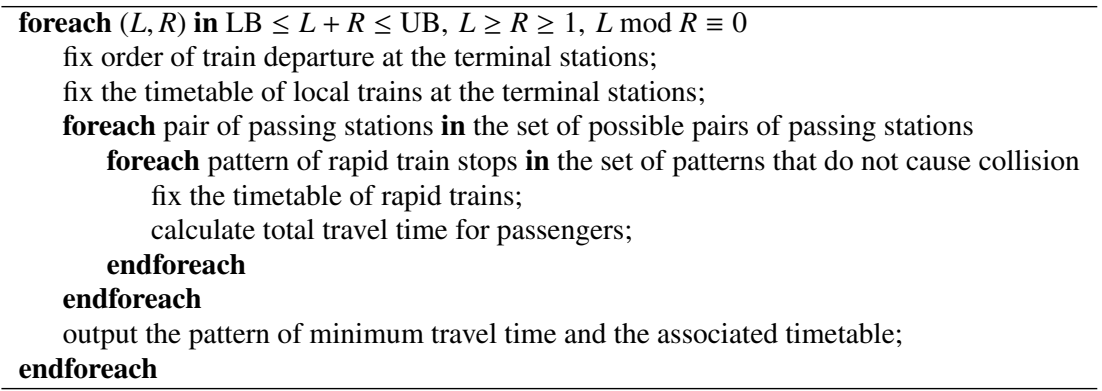

pattern of rapid train stops. Section 4.1 describes the basic framework of the algorithm, and Section 4.2 improves the algorithm to keep uniformity of the timetable.

\subsection{Basic framework}

The basic framework of the proposed algorithm is displayed in Table 1. In the following, the algorithm is explained in detail.

The algorithm takes input as follows:

- $L$ : the number of local trains operated per hour,

- $R$ : the number of rapid trains operated per hour,

- LB: the lower bound of $L+R$,

- UB: the upper bound of $L+R$,

- list of possible passing stations,

- matrix of travel duration between stations by a local train without being passed.

First, the outermost loop enumerates $(L, R)$ that satisfies $\mathrm{LB} \leq L+R \leq \mathrm{UB}, L \geq R \geq 1$ and $L \bmod R \equiv 0$, where the last condition is added by the fact that a timetable has a sixtyminute cycle (Condition (15)). For each $(L, R)$, we set the order of trains at the terminal stations as follows: the first train in an hour is a local train, the next is a rapid one, and the following $L / R-1$ trains are local ones; and that this pattern is repeated in the rest of the hour. Accordingly, Condition (13) is satisfied. Then, we determine the timetable of local trains. By Conditions (15) and (16), without loss of generality, one can set a timetable so that local trains depart from the terminal stations on the minute " 00 ." Then, the depart time of local trains at the terminal stations is " $00,60 / L, 2 \times 60 / L, \ldots,(L-1) \times 60 / L$."

Next, the middle loop enumerates pairs of passing stations, one for inbound and the other for outbound. As mentioned previously, in a railway line there are a few possible passing stations. Thus, the number of possible pairs of passing stations is not so large.

The innermost loop enumerates patterns of rapid train stops. The number of patterns of rapid train stops is $2^{n-4}$ for the fixed pair of passing stations, where $n$ is the number of stations in the railway line, because by Condition (8) rapid trains must stop at the both terminal stations and the passing stations for inbound and outbound trains; if the passing stations for inbound and outbound trains are identical, then the number is replaced with $2^{n-3}$. In addition, even for a pattern of rapid train stops is fixed, there still exists large degree of freedom about the timetable of rapid trains at the terminal stations. Hence, we have difficulty to examine all possibility at this stage.

For example, let $(L, R)$ be $(3,3)$. Then, inbound local trains depart from the origin station at " $00,20,40$," and the first rapid train should depart there at between " 02 " and " 18 " inclusive due to Condition (12). Next, for inbound trains, assume that: the passing station is located at 15-minutes distant from the origin station by a local train; in the pattern of rapid train stops considered now there are six skipped stations between the origin and passing stations. Under the assumption, the first local train arrive at the passing station at " 15 ," and thus the rapid train should arrive at the passing station at "17" or later. Hence, the rapid train should depart from the origin station " 08 " or later. In addition, the second local train departs from the origin 
station at "20." Therefore the rapid train can depart from the origin station at between " 08 " and " 18 " inclusive. This degree of freedom makes enumeration of all possibility hard.

However, for example, if the rapid train departs from the passing station at "18," it arrives at the passing station at "27." In such a case, the previous local train waits there too long, because the local train arrived there at " 15 " already; the local train leaves there at "29" or later, so the passengers on the local train stays there at least 14 minutes. This is definitely unacceptable situation.

Take the above discussion into consideration, in this study, we follow the simple principle: waiting time of local trains being passed should be as short as possible. From Condition (12), a local train passed by a rapid train should stay at the passing station at least four minutes. Therefore, we fix a timetable of rapid trains so that:

- a rapid train must arrive at the passing station just two minutes later than a previous local train arrived there,

- the local train must leave there just two minutes after the rapid train departed there. Accordingly, a local train passed by a rapid train stays at the passing station for just four minutes.

Return to the example. On this case, the first local train depart from the origin station at " 00 " and arrive at the passing station at " 15 ." Then, due to the principle, the rapid train should reach (and leave) the passing station at "17." Thus, the time when the rapid train depart from the origin station is uniquely fixed to "08."

Although allowing longer waiting time of a local train might reduce the total travel time of passengers, this principle makes enumeration much easier: fixing pattern of rapid train stops directly determines a timetable of rapid trains.

In general, the timetable of rapid trains is fixed as follows. Local trains depart from the terminal stations at " $00,60 / L, 2 \times 60 / L, \ldots,(L-1) \times 60 / L$." Let $m$ be the duration from the terminal station to the passing station by a local train, and there are $k$ skipped stations the terminal and passing stations the pattern of rapid train stops considered here. The time $t$ of departure of the first rapid train from the terminal station must satisfy $t+m-k=m+2$ and $2 \leq t \leq 60 / L-2$.

If $k \leq 60 / L-4$, we can create a timetable satisfying all conditions by letting $t=k+2$. In addition, the timetable of other $R-1$ rapid trains at the terminal station is fixed as " $t+$ $60 / R, t+2 \times 60 / R, \ldots, t+(R-1) \times 60 / R$."

If $k>60 / L-4$, which corresponds to patterns such that the number of rapid train stops between the origin and passing stations is too small, a rapid train could collide with the preceding local train even if the rapid train departs from the terminal station just two minutes earlier than the next local train. Accordingly, examining patterns with $k>60 / L-4$ does not make sense. These considerations speed up the process of enumerating patterns of rapid train stops.

Now each pattern of rapid train stops that satisfies $k \leq 60 / L-4$ corresponds to a timetable. As mentioned, the number of patterns of rapid train stops is $2^{n-3}$ or $2^{n-4}$. Hence, for a medium-scaled railway line (less than 30 stations), this algorithm runs in a practical computational time.

Finally, we calculate the total travel duration of passengers for each pattern of rapid train stops. The pattern of rapid train stops and the associated timetable that minimizes the total travel duration are output as the best solution for given input parameters.

\subsection{Modified algorithm}

In the previous subsection, we described the basic framework of the proposed algorithm. When $L=R$, the algorithm works well; local trains are operated at $60 / L$-minute intervals at each station. (In addition, rapid trains are also operated at $60 / R$-minute intervals.) However, in the case of $L>R \geq 1$, this uniformity of intervals for local trains is lost at stations after the passing station, even if the uniformity is held at the origin station. The reason is that some 


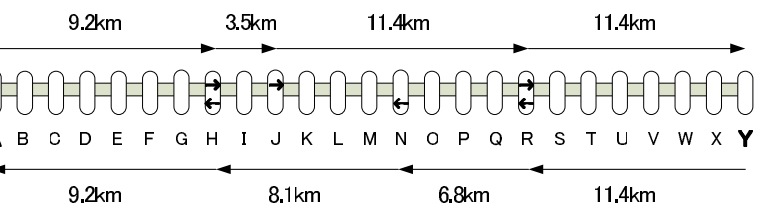

A: Kawasaki
E: Hirama
I: Musashi-Shinjōo
M: Shukugawara
Q: Yanokuchi
U: Bubaigawara
Y: Tachikawa

B: Shitte

F: Mukaigawara

J: Musashi-Mizonokuchi

$\mathrm{N}$ : Noborito

R: Inaginaganuma

V: Yaho

Y: Tachikawa

Fig. 1 JR Nambu line route map and possible passing stations

Table 2 Possible pairs of $(L, R)$

\begin{tabular}{cc}
\hline$L+R$ & $(L, R)$ \\
\hline 4 & $(3,1),(2,2)$ \\
5 & $(4,1)$ \\
6 & $(5,1),(4,2),(3,3)$ \\
7 & $(6,1)$ \\
8 & $(7,1),(6,2),(4,4)$ \\
9 & $(8,1),(6,3)$ \\
10 & $(9,1),(8,2),(5,5)$ \\
\hline
\end{tabular}

local trains are passed by a rapid train and the others are not.

Assume that $(L, R)$ is $(6,1)$ with local trains departing from the terminal station at " 00,10 , 20, 30, 40, 50" and rapid trains depart there at "05." At the passing station, the first rapid train passes the local train that departed from the terminal station at " 00 ." Then let us examine the timetable of a station such that: (1) no rapid train stops; (2) the station located after the passing station; (3) $m$-minutes distant from the terminal station by a local train without being passed. The timetable of local trains there becomes " $00+m+4,10+m, 20+m, 30+m, 40+m, 50+m$ (mod 60)," and the uniformity of intervals between local trains is lost.

We soften the loss of uniformity, when $L>R \geq 1$, by adding two minutes for the both preceding and subsequent local trains of the passed local train. Accordingly, the timetable is modified into " $00+m+4,10+m+2,20+m, 30+m, 40+m, 50+m+2(\bmod 60)$," in which uniformity is rather recovered. This is just a heuristic procedure; however, in the next section the computational experiments show that the procedure indeed produces better results compared to the results without this procedure when $L>R \geq 1$.

\section{Computational Experiments}

In this section, we describe the results of computational experiments. The experiments are performed for the JR Nambu line as an example. Section 5.1 introduces the JR Nambu line, and Section 5.2 describes the results of the experiments.

\subsection{JR Nambu line}

The JR Nambu line connecting Kawasaki Station (Kanagawa Prefecture) and Tachikawa Station (Tokyo Metropolis), is $35.5 \mathrm{~km}$ long. It links 25 stations including the terminal stations. The average distance between two adjacent stations is $1.42 \mathrm{~km}$ and that of passenger travel is $9.1 \mathrm{~km}$. It takes about 53 minutes by a local train between the two terminal stations, Kawasaki and Tachikawa.

Figure 1 portrays the route map and possible passing stations of the JR Nambu line. Stations $\mathrm{A}$ and $\mathrm{Y}$ in Fig. 1 are the terminal stations, Kawasaki and Tachikawa, respectively. Outbound trains go from left to right on the map. The stations indicated by arrow(s) are possible passing stations; a rapid train in the direction of the arrow can pass a local train in the same direction. As the figure shows, only Stations H (Musashi-Nakahara), J (Musashi- 
Table 3 Results of preliminary experiment

\begin{tabular}{ccccc}
\hline$L+R$ & $L$ & $R$ & time $_{4.1}(\mathrm{~m})$ & time $_{4.2}(\mathrm{~m})$ \\
\hline 4 & 3 & 1 & 21.54 & 21.48 \\
5 & 4 & 1 & 19.50 & 19.43 \\
6 & 5 & 1 & 18.26 & 18.17 \\
6 & 4 & 2 & 18.58 & 18.45 \\
7 & 6 & 1 & 17.41 & 17.33 \\
8 & 7 & 1 & 16.80 & 16.73 \\
8 & 6 & 2 & 16.89 & 16.74 \\
9 & 8 & 1 & 16.35 & 16.28 \\
9 & 6 & 3 & 16.37 & 16.16 \\
10 & 9 & 1 & 16.06 & 15.94 \\
10 & 8 & 2 & 16.02 & 15.90 \\
\hline
\end{tabular}

Mizonokuchi), N (Noborito), and R (Inagi-Naganuma) allow passing. Among these, Station J allows passing only for outbound trains and Station $\mathrm{N}$ only for inbound trains.

The station with the most boarding passengers is Station A (Kawasaki) with 187,147 on average per day, followed by Station Y (Tachikawa) with 158,068 on average per day; the fewest passengers board at Station K (Tsudayama) with 3,635 on average per day ${ }^{(6)}$.

As a few cases among railway lines in Tokyo area, the JR Nambu line currently provides no rapid train service. It runs six local trains per hour during daytime. However, the JR Nambu line operated rapid trains during 1969-1978, and it will resume in March 2011 for the first time in 33 years.

Our research is performed for the JR Nambu line as an example. Some reasons why we draw attention to this line include the following: (i) because no rapid train service is currently given there, this line is an interesting target to evaluate the effectiveness of operating rapid trains; (ii) because a couple of preceding studies conducted similar calculations for this line, our results could be compared with them.

Previous studies (3) and (5) have also examined assignment of rapid train stops on the JR Nambu line. These two papers reported the results in which Station $\mathrm{N}$ is assigned as the passing station for both inbound and outbound trains. However, Station $\mathrm{N}$ has multiple platforms only for the inbound direction. We consider this actual situation in the experiments described in the next section.

\subsection{Computational results}

For numerical experiments, we used OD data of passengers of the JR Nambu line (9:0017:00), extracted from Transportation Census of Urban Cities ${ }^{(7)}$. Four different OD datasets are available, and for each dataset we performed experiments. The number of passengers per hour of OD data I, II, III and IV are 7,700, 10,931, 13,098 and 16,329, respectively.

We set the upper bound UB and lower bound LB of $L+R$ as 4 and 10, respectively, because in the JR Nambu line trains are currently operated with $(L, R)=(6,0)$ in daytime. The number of possible $(L, R)$ is 15 , shown in Table 2 . There are $3 \times 3=9$ possible pairs of passing stations in the JR Nambu line (Stations H, N and R for inbound, Stations H, J and R for outbound; see Fig. 1).

First, we introduce the results of preliminary experiments that show the efficiency of the improvement mentioned in Section 4.2. In Table 3, using OD data IV, the columns "time 4.1 " and "time 4.2 " are the average travel time for passengers without and with the improvement, respectively. Clearly, the latter results are better. For OD data I, II and III, the same results were obtained. Accordingly, in the following all experiments, we apply the procedure to the algorithm.

Table 4 shows that, for each OD data, how many times each station is selected for a rapid train stop out of 15 possible $(L, R)$ in Table 2. Tables $6,7,8$ and 9 display the results of experiments for OD data I, II, III and IV, respectively. In these tables, "time (m)" stands for the average duration of travel per passenger (minute); " $\rightarrow$ " and " $\leftarrow$ " are the optimal passing 
Table 4 Distribution of rapid train stops

\begin{tabular}{|c|c|c|c|c|c|c|c|c|c|c|c|}
\hline & & C D & $\mathrm{E} F \quad \mathrm{~F} \quad \mathrm{H} \quad \mathrm{I}$ & K L & $\mathrm{M} \mathrm{N}$ & $0 \mathrm{P}$ & $\mathrm{Q} R$ & $S \mathrm{~T} U \mathrm{UV}$ & $\overline{\mathrm{V}}$ & $\mathrm{X} \mathrm{Y}$ & erage \\
\hline$D$ data I & 15 & 1515 & $0 \quad 41515$ & 03 & 3015 & 312 & 17 & 1151515 & & 1101 & 14 \\
\hline & 15 & 41115 & $\begin{array}{lll}9 & 0\end{array}$ & $\begin{array}{ll}0 & 0\end{array}$ & 015 & 312 & 315 & 0 & 3 & 415 & 14.3 \\
\hline & 15 & $\begin{array}{lll}0 & 8 & 15\end{array}$ & 1515 & $\begin{array}{ll}0 & 3\end{array}$ & 3015 & 814 & 015 & 01 & & 0 & 16.2 \\
\hline $\mathrm{O}$ & 15 & 1815 & 101 & 03 & $3 \quad 015$ & 412 & 015 & 01515 & 0 & 01015 & 15.2 \\
\hline total & 60 & 542603 & 560 & $0 \quad 9$ & 906 & 850 & 452 & 1586033 & & 42660 & 14.9 \\
\hline
\end{tabular}

Table 5 Comparison with the previous research ${ }^{(5)}$

\begin{tabular}{|c|c|c|c|c|}
\hline research & $L+R$ & $L R$ & $\rightarrow \leftarrow$ & time $(\mathrm{m})$ \\
\hline this study & 6 & 42 & $\mathrm{~J} \mathrm{~N}$ & 18.45 \\
\hline Hiroto $^{(5)}$ & 6 & 42 & $\mathrm{~N} \mathrm{~N}$ & 18.50 \\
\hline this study (NN) & 6 & 42 & $\mathrm{~N} \mathrm{~N}$ & 18.45 \\
\hline this study & 8 & 62 & R N & 16.80 \\
\hline Hiroto $^{(5)}$ & 8 & 62 & $\mathrm{~N} \mathrm{~N}$ & 16.79 \\
\hline this study $(\mathrm{NN})$ & 8 & 62 & $\mathrm{~N} \mathrm{~N}$ & 16.74 \\
\hline this study & 8 & 44 & R R & 16.90 \\
\hline Hiroto $^{(5)}$ & 8 & 44 & $\mathrm{~N} \mathrm{~N}$ & 16.91 \\
\hline this study (NN) & 8 & 44 & $\mathrm{~N} \mathrm{~N}$ & 16.86 \\
\hline
\end{tabular}

stations for outbound and inbound trains, respectively; “*” indicates rapid train stops in each optimal assignment; "\#stops" means the number of rapid train stops. For reference, these tables include the cases of $R=0$ (operating only local trains).

We infer the following outcome from the experiments.

- Optimal assignment of rapid train stops mainly depends on OD data rather than the values of $L$ and $R$. For instance, regardless of $(L, R)$, Station $\mathrm{F}$ should be a rapid train stop for OD data III and IV, and should not be for OD data I and II. Similarly, regardless of $(L, R)$, Station V should be a rapid train stop for OD data I and III, and should not be for OD data II and IV.

- Several stations are probable candidates for rapid train stops for all OD data: Stations D, G, H, J, N, T and U. Similarly, from the results, there are some stations rapid trains should skip: Stations B, K, L, M, Q, S and W.

- In a couple out of 60 settings (four OD data and 15 possible $(L, R)$ ), the number of rapid train stops is 12 , and in another couple of settings the number is 18 . In all other 56 settings, the numbers of rapid train stops are between 13 and 17.

- Station $\mathrm{H}$ is of small importance as a passing station.

- For OD data III and IV, Station R is of great importance for a passing station.

- For $L+R \leq 7$, rapid train service does not improve the total travel duration of passengers, compared to operating only local trains; for $L+R \geq 8$, rapid train service slightly improves of the total travel duration of passengers.

Table 5 shows the comparison between the previous study by Hiroto ${ }^{(5)}$ and the obtained results. (Here we note that although Matsumura ${ }^{(3)}$ dealt with the JR Nambu line, his results cannot be compared with ours because the total travel duration of passengers was not reported in his results.) The OD data used in Hiroto ${ }^{(5)}$ is OD data IV of 16,329 passengers. In his study, the pair of passing stations is fixed to $(\mathrm{N}, \mathrm{N})$, where the first element is the passing station for outbound trains and the second one is for inbound. In this paper, a pair of passing stations is variable, but $(\mathrm{N}, \mathrm{N})$ is not allowed due to the actual situation of platforms (see Fig. 1). In the table, the row "this study" displays our results where possible passing stations are provided as Fig. 1; "Hiroto(5)" does the results by (5). For comparison purpose, we also conducted experiments not only the cases where $(\mathrm{N}, \mathrm{N})$ is not allowed, but also ones where the passing stations are fixed to (N, N) for OD data IV; "this study (NN)" shows the results where both passing stations are set to Station N. For $(L, R)=(4,2)$, our result uses Station J as a passing station for inbound trains instead of Station $\mathrm{N}$, and the total travel time is reduced. For $(L, R)=(6,2)$ and $(4,4)$, his results and ours are comparable. It means that, even without using $(\mathrm{N}, \mathrm{N})$ as passing stations, a timetable as good as the results with $(\mathrm{N}, \mathrm{N})$ can be obtained. 
Table 6 Results for OD data I

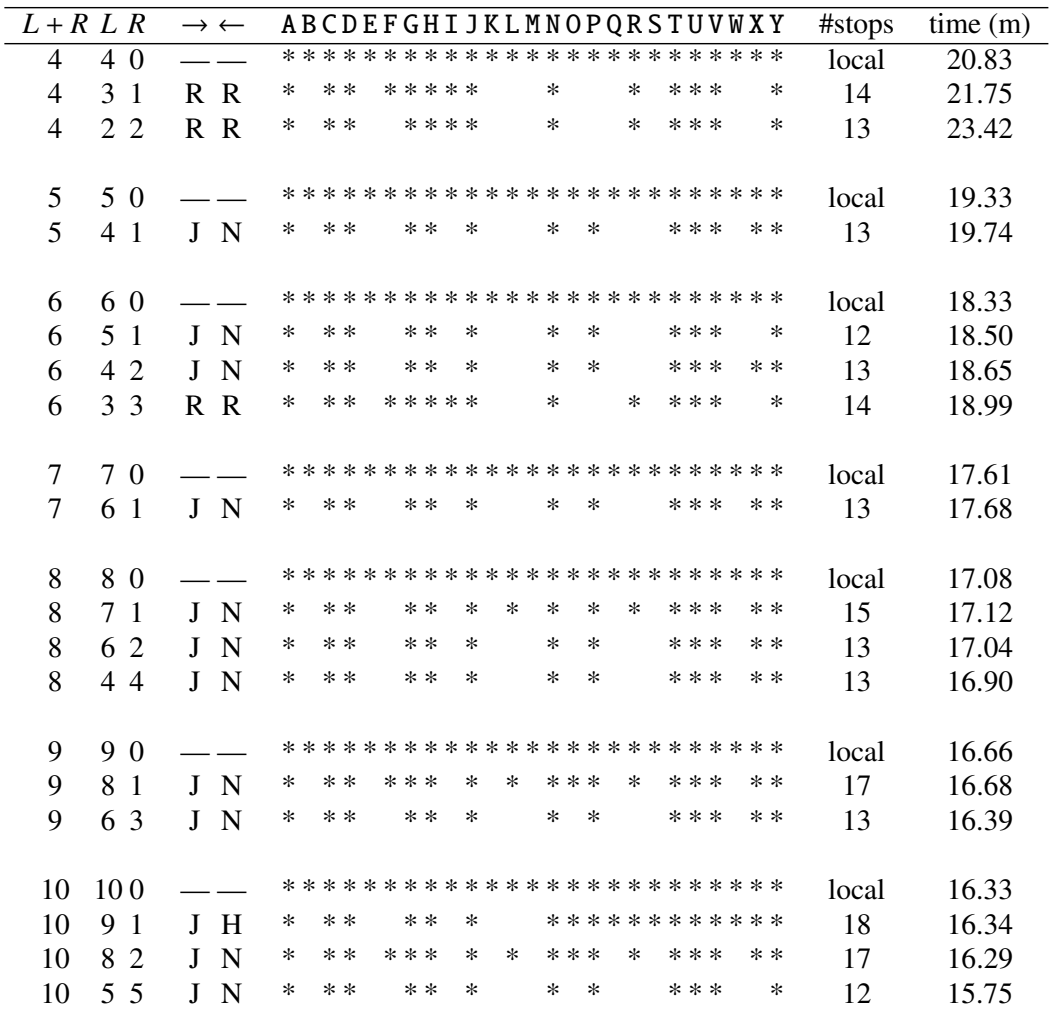

Table 7 Results for OD data II

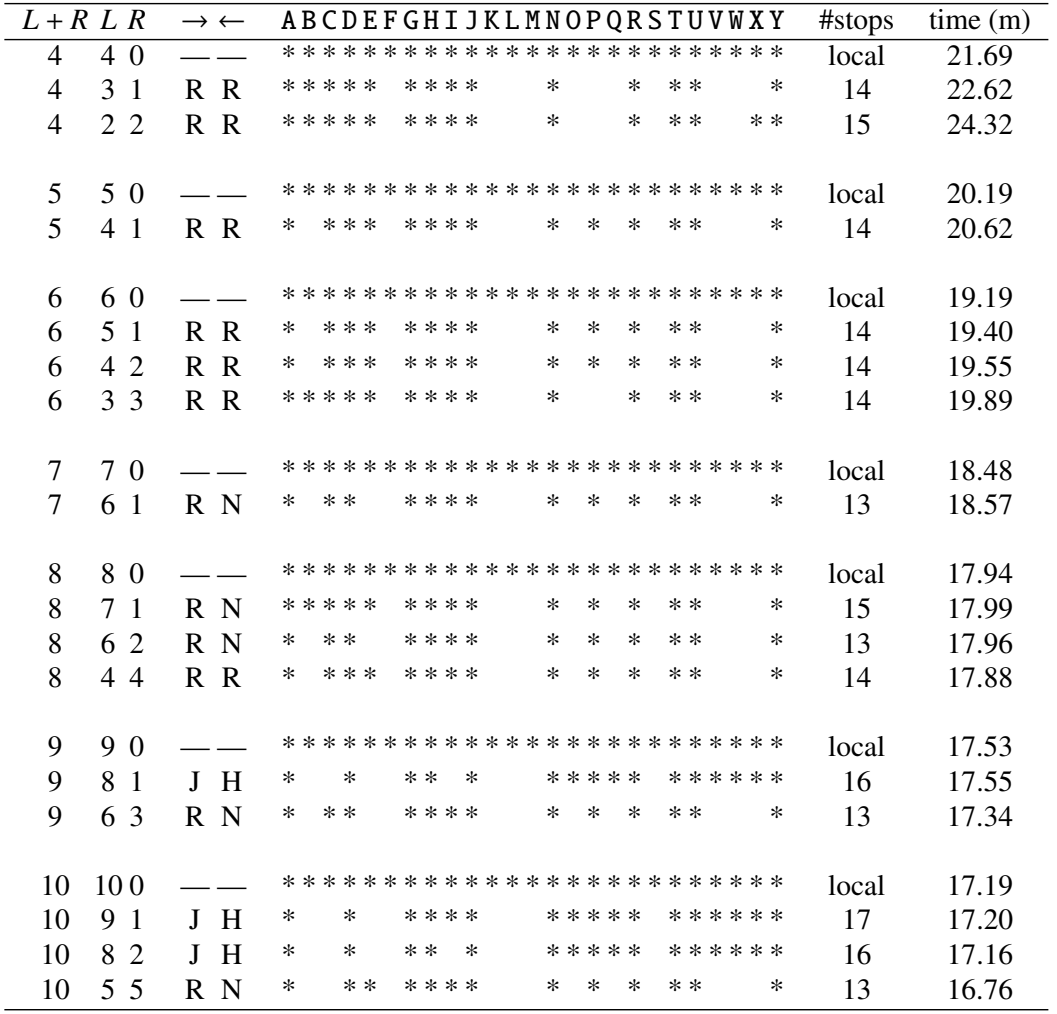

As shown above, these experiments can provide quantitative analysis, in addition to a qualitative one, to determine the rapid train stops, passing stations and associated timetables.

\section{Conclusion}

In this paper, we proposed an enumeration-based algorithm for determining optimal as- 
Table 8 Results for OD data III

\begin{tabular}{|c|c|c|c|c|c|c|c|c|c|c|}
\hline$L+R$ & $L R$ & $\rightarrow \leftarrow$ & & A B CDEFGH I J K L M & NOP & QR & S T UVWX & & \#stops & time $(\mathrm{m})$ \\
\hline 4 & 40 & -1 & & $* * * * * * * * * * * * *$ & $* * *$ & $* *$ & $* * * * * *$ & & local & 19.37 \\
\hline 4 & 31 & R I & $\mathrm{R}$ & $* \quad * * * * * * * *$ & $* *$ & $*$ & $* * *$ & $* *$ & 16 & 20.49 \\
\hline 4 & 22 & $\mathrm{R} \mathrm{I}$ & $\mathrm{R}$ & $* \quad * * * * * * * *$ & $*$ & $*$ & $* * \quad *$ & $* *$ & 15 & 22.53 \\
\hline 5 & 50 & -1 & - & $* * * * * * * * * * * * *$ & $* * *$ & $* *$ & $* * * * * *$ & $* *$ & local & 17.87 \\
\hline 5 & 41 & $\mathrm{R}$ & $\mathrm{R}$ & $* \quad * * * * * * * *$ & $* *$ & $*$ & $* * *$ & $*$ & 16 & 18.42 \\
\hline 6 & 60 & -1 & - & $* * * * * * * * * * * * *$ & $* * *$ & $* *$ & $* * * * * *$ & $* *$ & local & 16.87 \\
\hline 6 & 51 & $\mathrm{R}$ & $\mathrm{R}$ & $* \quad * * * * * * * *$ & $* *$ & $*$ & $* * *$ & * & 16 & 17.17 \\
\hline 6 & 42 & $\mathrm{R} \mathrm{I}$ & $\mathrm{R}$ & $* \quad * * * * * * * *$ & $* *$ & * & $* * *$ & * & 16 & 17.47 \\
\hline 6 & 33 & $\mathrm{R} \mathrm{I}$ & $\mathrm{R}$ & $* \quad * * * * * * * *$ & $* *$ & $*$ & $* * *$ & * & 16 & 18.04 \\
\hline 7 & 70 & -1 & - & $* * * * * * * * * * * * *$ & $* * *$ & $* *$ & $* * * * * *$ & $* *$ & local & 16.16 \\
\hline 7 & 61 & $\mathrm{R} \mathrm{I}$ & $\mathrm{R}$ & $* * * * * * *$ & $* * *$ & $*$ & $* * *$ & $*$ & 16 & 16.33 \\
\hline 8 & 80 & & - & $* * * * * * * * * * * * *$ & $* * *$ & $* *$ & $* * * * * *$ & $* *$ & local & 15.62 \\
\hline 8 & 71 & $\mathrm{R} \mathrm{I}$ & & $* * * * * * *$ & $* * *$ & * & $* * *$ & * & 16 & 15.73 \\
\hline 8 & 62 & $\mathrm{R}$ & $\mathrm{R}$ & $* * * * * * *$ & $* * *$ & * & $* * *$ & * & 16 & 15.80 \\
\hline 8 & 44 & R I & $\mathrm{R}$ & $* \quad * * * * * * * *$ & $* *$ & $*$ & $* * *$ & $*$ & 16 & 15.97 \\
\hline 9 & 90 & -1 & - & $* * * * * * * * * * * * *$ & $* * *$ & $* *$ & $* * * * * *$ & $* *$ & local & 15.21 \\
\hline 9 & 81 & $\mathrm{R} \mathrm{I}$ & $\mathrm{R}$ & $* * * * * * * \quad *$ & $* * *$ & * & $* * *$ & * & 17 & 15.28 \\
\hline 9 & 63 & R I & $\mathrm{R}$ & $* * * * * * *$ & $* * *$ & $*$ & $* * *$ & $*$ & 16 & 15.26 \\
\hline 10 & 100 & -1 & - & $* * * * * * * * * * * * *$ & $* * *$ & $* *$ & $* * * * * *$ & $* *$ & local & 14.87 \\
\hline 10 & 91 & $\mathrm{R} \mathrm{I}$ & $\mathrm{R}$ & $* \quad * * * * * * * * \quad *$ & $* * *$ & $*$ & $* * *$ & $*$ & 18 & 14.92 \\
\hline 10 & 82 & $\mathrm{R}$ & $\mathrm{R}$ & $* * * * * * * \quad *$ & $* * *$ & $*$ & $* * *$ & $*$ & 17 & 14.94 \\
\hline 10 & 55 & $\mathrm{R}$ & $\mathrm{R}$ & $* * * * * * *$ & $* * *$ & * & $* * *$ & $*$ & 16 & 14.85 \\
\hline
\end{tabular}

Table 9 Results for OD data IV

\begin{tabular}{|c|c|c|c|c|c|c|c|c|c|c|c|}
\hline \multicolumn{2}{|c|}{$L+R L R$} & \multirow{2}{*}{\multicolumn{2}{|c|}{$\begin{array}{l}\rightarrow \leftarrow \\
-\leftarrow\end{array}$}} & \multicolumn{6}{|c|}{ A B CDEFGH I J K L M N OPQR S T UVWXY } & \multirow{2}{*}{$\begin{array}{c}\text { \#stops } \\
\text { local }\end{array}$} & \multirow{2}{*}{$\begin{array}{c}\text { time }(\mathrm{m}) \\
20.42\end{array}$} \\
\hline 4 & 40 & & & $* * * * * * * * * * *$ & $* * *$ & $* * * *$ & $* *$ & $* * *$ & $* * *$ & & \\
\hline 4 & 31 & $\mathrm{R}$ & $\mathrm{R}$ & $* \quad * * * * * * * *$ & & $*$ & $*$ & $* *$ & $* *$ & 15 & 21.48 \\
\hline 4 & 22 & $\mathrm{R}$ & $\mathrm{R}$ & $* * * * \quad * * * * *$ & & $*$ & $*$ & $* *$ & $* *$ & 15 & 23.41 \\
\hline 5 & 50 & - & - & $* * * * * * * * * * *$ & $* * *$ & $* * * *$ & $* *$ & $* * *$ & $* * *$ & local & 18.92 \\
\hline 5 & 41 & $\mathrm{R}$ & $\mathrm{R}$ & $* \quad * * * * * * * *$ & & $* \quad *$ & $*$ & $* *$ & $* *$ & 16 & 19.43 \\
\hline 6 & 60 & - & - & $* * * * * * * * * * *$ & $* * *$ & $* * * *$ & $* *$ & $* * *$ & $* * *$ & local & 17.92 \\
\hline 6 & 51 & $\mathrm{R}$ & $\mathrm{R}$ & $* \quad * * * * * * * *$ & & $* \quad *$ & $*$ & $* *$ & $*$ & 15 & 18.20 \\
\hline 6 & 42 & $\mathrm{R}$ & $\mathrm{R}$ & $* \quad * * * * * * * *$ & & * * & $*$ & $* *$ & $* *$ & 16 & 18.45 \\
\hline 6 & 33 & $\mathrm{R}$ & $\mathrm{R}$ & $* \quad * * * * * * * *$ & & $*$ & $*$ & $* *$ & $* *$ & 15 & 18.95 \\
\hline 7 & 70 & - & — & $* * * * * * * * * * *$ & $* * *$ & $* * * *$ & $* *$ & $* * *$ & $* * *$ & local & 17.21 \\
\hline 7 & 61 & $\mathrm{R}$ & $\mathrm{N}$ & $* \quad * * * * *$ & & $* \quad *$ & $*$ & $* *$ & $* *$ & 14 & 17.36 \\
\hline 8 & 80 & - & 一 & $* * * * * * * * * * *$ & $* *$ & $* * * *$ & $* *$ & $* * *$ & $* * *$ & local & 16.67 \\
\hline 8 & 71 & $\mathrm{R}$ & $\mathrm{N}$ & $* * * * * * *$ & & $* * *$ & $*$ & $* *$ & $*$ & 15 & 16.76 \\
\hline 8 & 62 & $\mathrm{R}$ & $\mathrm{N}$ & $* \quad * * * * *$ & & $* \quad *$ & $*$ & $* *$ & $* *$ & 14 & 16.80 \\
\hline 8 & 44 & $\mathrm{R}$ & $\mathrm{R}$ & $* \quad * * * * * * * *$ & & $* \quad *$ & $*$ & $* *$ & $* *$ & 16 & 16.90 \\
\hline 9 & 90 & - & - & $* * * * * * * * * * *$ & $* * *$ & $* * * *$ & $* *$ & $* * *$ & $* * *$ & local & 16.26 \\
\hline 9 & 81 & $\mathrm{R}$ & $\mathrm{R}$ & $* * * * * * *$ & $*$ & $* * *$ & $*$ & $* *$ & * & 16 & 16.31 \\
\hline 9 & 63 & $\mathrm{R}$ & $\mathrm{N}$ & $* \quad * * * * *$ & & $* *$ & $*$ & $* *$ & $* *$ & 14 & 16.23 \\
\hline 10 & 100 & - & 一 & $* * * * * * * * * * *$ & $* *$ & $* * * *$ & $* *$ & $* * *$ & $* * *$ & local & 15.92 \\
\hline 10 & 91 & $\mathrm{R}$ & $\mathrm{R}$ & $* \quad * * * * * * * *$ & $*$ & $* * *$ & $*$ & $* *$ & $*$ & 17 & 15.96 \\
\hline 10 & 82 & $\mathrm{R}$ & $\mathrm{R}$ & $* * * * * * *$ & $*$ & $* * *$ & $*$ & $* *$ & $*$ & 16 & 15.95 \\
\hline 10 & 55 & $\mathrm{R}$ & $\mathrm{N}$ & $* \quad * * * * *$ & & $* \quad *$ & $*$ & $* *$ & $* *$ & 14 & 15.77 \\
\hline
\end{tabular}

signment of rapid train stops and the associated timetable that minimize total travel time for passengers. Using the proposed algorithm, we determined optimal assignment of rapid train stops for the JR Nambu line, which is a medium-sized railway line with 25 stations in Japan.

The procedure introduced here does not allow railway lines with branches and operations 
over part of a line. The modifications of our present procedure to accommodate the items above and for large-scaled lines are future works.

\section{References}

(1) Suzuki, S. and Ishizuka, Y., An Approximation Algorithm for the Problem of Determination of Express Train Stops (in Japanese), The Institute of Statistical Mathematics Cooperative Research Report, Vol. 61, (1994), pp. 154-158.

( 2 ) Futami, S., Matsumura, T. and Suzuki, S., Problems of Deciding Express Train Stops (in Japanese), Proceedings of 2001 Spring Conference of Operations Research Society of Japan, (2001), pp. 84-85.

( 3 ) Matsumura, T., A Study on Setting Method of Express Trains (in Japanese), Communications of the Operations Research Society of Japan, Vol. 48, No. 1 (2003), pp. 50-51.

( 4 ) Katori, T., Takahashi, Y. and Izumi, T., To Shorten Total Trip Time for Passengers by Rapid Trains Operation: Determination of the Stations where Rapid Train Stops or Passes to Local Trains Used Genetic Algorithm (in Japanese), The Transactions of the Institute of Electrical Engineers of Japan, Vol. 125-D, No. 4 (2005), pp. 305-312.

( 5 ) Hiroto, A., Determination of Rapid Train Stops and Construction of Optimal Diagram (in Japanese), Bachelor Thesis, Faculty of Science and Technology, (2009), Keio University.

( 6 ) East Japan Railway Company, "Number of Passengers in Stations (in Japanese)", East Japan Railway Company, (online), available from http://www.jreast.co.jp/passenger/ index.html (accessed as of 2011-10-27).

( 7 ) Ministry of Land, Infrastructure, Transport and Tourism, Transportation Census of Urban Cities (in Japanese), No. 10, (2005), Ministry of Land, Infrastructure, Transport and Tourism, Tokyo. 